\title{
RECHERCHE DES STAPHYLOCOQUES PATHOGÈNES DANS LE LAIT ET LES PRODUITS LAITIERS
}

\author{
par \\ G. THIEULIN, D. BASILLE, J. PANTALEON, \\ R. ROSSET, Y. GANDON et A. PETIT
}

Dans le cadre des missions qui lui sont dévolues, le vétérinaire sanitaire est intéressé à l'inventaire de la flore bactérienne du lait et des produits laitiers en tant que moyen d'atteindre les objectifs suivants :

- Détection de cas de maladies ou d'enzooties mettant en péril la vie de l'animal, le rendement de la production et la qualité des produits.

- Découverte de foyers de maladies animales transmissibles à l'homme ou susceptibles de rendre nocifs pour l'homme les aliments provenant des animaux malades.

- Dépistage de porteurs de germes parmi le personnel ayant participé à la préparation et à la manipulation des produits contaminés.

- Retrait de la consommation, des aliments contenant des germes pathogènes pour l'homme.

- Vérification de la présence des éléments de la flore normale nécessaire à l'évolution du produit et faisant partie des qualités substantielles de celui-ci.

- Détection d'éléments étrangers à la flore normale et témoignant que des fautes techniques ont été commises au cours de la préparation ou de la conservation de la denrée.

Les bactéries dont l'identification n'a pas de rapport avec l'obtention des résultats ci-dessus considérés, ne sauraient intéresser l'hygiéniste que comme éléments purement numériques d'une flore totale dont le dénombrement global est susceptible de fournir une base d'appréciation de la valeur du produit : c'est dire que l'identification spécifique de ces bactéries ne présente pas d'utilité, il suffit de pouvoir les compter.

Plusieurs des considérations ci-dessus mentionnées entrent en ligne de compte pour attirer notre attention sur la détection et le dénombrement des staphylocoques du lait et des produits laitiers, que ce soit au regard de la santé animale et de l'économie agricole, ou qu'il s'agisse, dans le domaine de l'hygiène alimentaire, de 
contrôler la salubrité et la qualité des denrées d'origine animale destinées à la consommation humaine.

On voit donc que, si la classification des microcoques et staphylocoques constitue pour le taxonomiste, selon l'expression de S. T. Cowan, une "introduction au Chaos", le problème se pose par ailleurs en termes beaucoup plus simples puisque ce qui nous importe ici c'est seulement de savoir effectuer, sur des bases sûres et pratiques, la discrimination des staphylocoques doués de pouvoir pathogène pour l'homme et pour l'animal.

Cette détermination est possible. Elle est même devenue de plus en plus facile au fil des progrès d'une technique dont les principales étapes ont été marquées par les découvertes suivantes :

\section{Pouvoir pathogène}

Le pouvoir pathogène de certaines espèces de staphylocoques est dû à la production d'une exotoxine également nocive pour les animaux et pour l'homme.

\section{Coagulation du plasma}

Ea sécrétion de toxine va de pair avec la production d'un enzyme capable de coaguler le plasma. En particulier, les staphylocoques responsables d'intoxications alimentaires causées par la consommation de produits laitiers ou de lait sont toujours des souches à coagulase positive ainsi que l'a montré Newman.

\section{Halophilie}

Koch a montré que les staphylocoques se développaient sans restriction dans un milieu contenant une forte proportion de chlorure de sodium capable d'inhiber la croissance de la plupart des autres bactéries (principe du milieu liquide d'enrichissement).

\section{Attaque du mannitol}

Dans un milieu gélosé hypersalé contenant du d-mannitol et du rouge de phénol, les staphylocoques à coagulase positive forment des colonies vigoureuses qui s'entourent d'une zone dans laquelle l'indicateur est viré au jaune (principe du milieu d'isolement et d'identification dérivé des observations de Chapman). Mac Culloch a vu que les colonies de staphylocoques agents de la mammite de la vache appartenaient au type ci-dessus décrit.

La gélose mannitée hypersalée, commercialisée sous forme de milieu composé déshydraté (Mannitol Salt Agar), a été longtemps le milieu le plus employé et demeure encore couramment utilisée. 
Cependant, dans bien des cas, l'interprétation des résultats pose des problèmes : en effet, les deux critères d'identification des colonies de staphylocoques à coagulase positive, à savoir le développement eugonique des colonies caractéristiques et le virage du rouge de phénol autour d'elles, peuvent être mis en échec, du fait de la présence d'une flore abondante de microcoques et de bacilles dans les cultures. A cause du surpleuplement, il arrive que les colonies de staphylocoques à coagulase positive n'acquièrent qu'un développement restreint, guère plus important que celui des autres colonies. Par ailleurs, les zones jaunes produites par les germes mannitol-positifs s'étalent largement autour des colonies. Il en résulte que des colonies de germes mannitol-négatifs mélangées à quelques colonies mannitol-positives peuvent se trouver incluses dans des plages jaunes et être comptées pour des colonies de staphylocoques pathogènes dont, morphologiquement, elles ne se distinguent pas nettement.

Pour prévenir ce risque, il devient nécessaire de soumettre les colonies suspectes à une épreuve enzymatique de confirmation, la culture sur gélose mannitée hypersalée n'étant plus considérée alors que comme un test de présomption.

Même si, pour le test de confirmation, on substitue à l'épreuve de la coagulase celle, beaucoup plus commode à exécuter, de la désoxyribonucléase comme semble l'autoriser le taux de concordance très élevé que nous avons constaté entre les deux épreuves (voir ci-après), la méthodé ainsi complétée reste peu compatible avec les impératifs de simplicité et de rapidité du contrôle de routine. On aura une idée du nombre de manipulations fastidieuses imposées par ce " passage au crible " des colonies suspectes d'après le "déchet" que nous avons obtenu en soumettant à l'épreuve de la désoxyribonucléase 423 colonies suspectes prélevées dans des culture sur gélose mannitée hypersalée (Mannitol Salt Agar, Difco). Ces 423 colonies se répartissaient, quant à l'origine, comme suit :

- 124 colonies provenaient de cultures issues d'échantillons de laits crus individuels prélevés au pis de la vache en vue du dépistage de la mammite staphylococcique ou du contrôle après traitement.

- 223 colonies étaient dérivées d'échantillons de laits crus de mélange restreint (étables de 6 à 15 vaches), prélevés en vue du contrôle hygiénique au stade de la vente direete au consommateur.

- 66 colonies provenaient de fromages frais, pour la plupart de fabrication domestique et non-pasteurisés, ou sous-pasteurisés ; (l'obtention de colonies suspectes à partir de fromages frais à phosphatase négative est un résultat que nous n'avons rencontré que très exceptionnellement et qui a toujours été démenti par l'épreuve de la désoxyribonucléase).

- 10 colonies provenaient de crèmes fraîches non pasteurisées. 
Nous avons enregistré les résultats suivants :

\begin{tabular}{|c|c|c|}
\hline $\begin{array}{c}\text { Origine } \\
\text { des eultures }\end{array}$ & $\begin{array}{c}\text { Nombre de colonies } \\
\text { suspectes testées }\end{array}$ & $\begin{array}{c}\text { p. cent de colonies } \\
\text { DNase + }\end{array}$ \\
\hline Laits individuels $\ldots \ldots \ldots \ldots \ldots$ & 124 & 50 \\
\hline Laits de mélange restreint .... & 223 & 44 \\
\hline Fromages frais $\ldots \ldots \ldots \ldots \ldots \ldots$ & 66 & 38 \\
\hline Crèmes fraîches $\ldots \ldots \ldots \ldots \ldots$ & 10 & 60 \\
\hline Total ............... & 423 & 45 \\
\hline
\end{tabular}

Ces résultats accusent un " déchet 》 de 55 p. 100 sur les colonies suspectes soumises au contrôle. Il est à noter, par ailleurs, que la proportion de colonies non confirmées est très variable d'une culture à l'autre, allant de 0 à $100 \mathrm{p}$. 100 . Il est évident qu'un tel manque de spécificité joint à l'impossibilité de faire jouer la règle de probabilité dans l'évaluation du "déchet", impose le recours systématique à une épreuve de contrôle de toute tentative de dénombrement des colonies de staphylocoques pathogènes sur gélose mannitée hypersalée.

\section{Dépolymérisation de l'acide désoxyribonucléique}

Pour le contrôle des colonies suspectes, on a d'abord fait appel à l'épreuve de la coagulase. Puis, après avoir constaté l'existence d'une corrélation étroite entre la production de coagulase et celle d'autres diastases, on a proposé de baser l'identification des souches de staphylocoques pathogènes sur la mise en évidence de la phosphatase acide, ou de la désoxyribonucléase (DNase). Cette dernière épreuve nous a semblé la plus commode : exécutée par la méthode d'ensemencement "à la touche " elle permet de vérifier simultanément une douzaine de colonies dans une seule boite de Pétri contenant la gélose spéciale (tryptose $20 \mathrm{~g}$; acide désoxyribonucléique $2 \mathrm{~g}$; ClNa $5 \mathrm{~g}$; agar $15 \mathrm{~g}$; eau distillée 1 litre) qui peut être préparée en faisant dissoudre $42 \mathrm{~g}$ de milieu préparé déshydraté du commerce (DNase Test Agar, Difco) dans un litre d'eau distillée. Après incubation de 18 à $24 \mathrm{H}$ à $37^{\circ}$, on couvre la surface de la culture d'une solution normale d'acide chlorhydrique : les plages correspondant au développement de souches DNase-positives apparaissent entourées d'une auréole transparente dont la largeur est au moins égale au diamètre de la plage microbienne circonscrite. On peut remplacer la solution d'acide chlorhydrique par une solution aqueuse de bleu de toluidine à 0.1 p. 100 : «les spots» correspondant au développement de souches DNase-positives s'entourent alors d'une zone rose-carmin.

Nous avons vérifié le taux de concordance entre les résultats des épreuves de la coagulase et de la DNase en exécutant les deux 
tests conjointement sur 200 colonies suspectes prélevées sur des cultures issues d'échantillons de lait et de produits laitiers. Pour ces prélèvements nous avons sélectionné les cultures sur gélose mannitée hypersalée les plus faciles à déchiffrer, en écartant les cultures surpeuplées. Nous avons enregistré les résultats suivants :

\begin{tabular}{ccc}
\hline \hline $\begin{array}{c}\text { Type de résultat } \\
\text { Coagulase }\end{array}$ & DNase & p. cent colonies \\
(sur 200)
\end{tabular}

Ces chiffres font apparaître un taux de concordance de 96 p. 100 entre les résultats des deux épreuves.

\section{Milieux au tellurite de potassium et au glycocolle}

En dépit de la simplification apportée par l'épreuve de la DNase, l'obligation de contrôler, par une épreuve enzymatique, les colonies développées sur gélose mannitée hypersalée demeurait une servitude fort gênante. C'est pourquoi les recherches s'orientèrent vers la mise au point de milieux plus sélectifs et différentiels : le milieu recherché devait permettre la.croissance, sans restriction de tous les staphylocoques à coagulase positive en inhibant au maximum les autres germes et en assurant un polymorphisme suffisant pour que les colonies de staphylocoques à coagulase positive pussent être reconnues sans confusion possible et sans nécessité de recourir à une épreuve de confirmation.

Ce fut tout d'abord le tellurite glycine agar de Zebovitz, Evans et Niven qui avait l'inconvénient d'exercer une sélection draconnienne n'épargnant même pas certaines souches de staphylocoques authenquement positifs à l'épreuve de la coagulase.

Par de nombreux tâtonnements, plusieurs bactériologistes s'efforcèrent d'améliorer ce milieu. Ces tentatives ont abouti en dernier lieu au milieu de Baird-Parker qui est bien près d'atteindre tous les objectifs ci-dessus mentionnés.

Dans une précédente étude, consacrée à la recherche et au dénombrement des staphylocoques présumés pathogènes dans les produits alimentaires (1) nous avions constaté que la culture directe sur milieu de Baird-Parker permettait la mise en évidence des staphylocoques à coagulase positive des produits carnés dans les meilleures conditions de commodité et de rapidité et avec les plus sûres garanties de fidélité et de sensibilité. Par contre, nous faisions quelques réserves en ce qui concerne l'application de cette méthode au dénombrement des mêmes germes dans le lait et les

(1) Annales de l'Institut Pasteur de Lille, 1964, 15-157-164. 
produits laitiers. Cependant, notre expérience en la matière étant, à l'époque, très courte, nous avions remis à plus tard de nous prononcer sur la validité de la méthode dans ce secteur d'application.

Nous exposons ci-après les résultats de l'emploi du milieu de Baird-Parker dans les conditions de l'inspection de routine :

1) des laits crus individuels, prélevés aseptiquement au pis de la vache en vue du dépistage de la mammite ou du contrôle des traitements ;

2) des laits crus de mélange restreint (étables de 6 à 15 vaches), vendus directement au consommateur;

3) des fromages frais (en grande majorité de fabrication domestique et non-pasteurisés, les fromages frais pasteurisés du commerce s'étant révélés régulièrement exempts de staphylocoques à coagulase positive, à l'exception de certains échantillons de Fontainebleau obtenus par mélange de lait caillé pasteurisé avec de la crème non pasteurisée, ainsi qu'en témoignait une phosphatase non totalement inactivée ;

4) de quelques échantillons de crème non-pasteurisée ou incorrectement pasteurisée.

\section{Préparation du milieu de base}

Faire dissoudre à chaud : tryptone $10 \mathrm{~g}$; Lab-Lemco (Oxoïd) $5 \mathrm{~g}$; extrait de levure $1 \mathrm{~g}$; chlorure de lithium $5 \mathrm{~g}$; agar $20 \mathrm{~g}$, dans eau distillée q.s. pour $1000 \mathrm{ml}$.

Ajuster à $p \mathrm{H} \mathrm{7,2;} \mathrm{distribuer} \mathrm{par} \mathrm{fractions} \mathrm{de} 83 \mathrm{ml}$ en fioles jaugées à $100 \mathrm{ml}$; stériliser à l'autoclave à $120^{\circ} \mathrm{C}$ pendant 15 minutes.

\section{Préparation des solutions filtrées}

Stériliser par filtration sur disques Seitz les solutions aqueuses suivantes : glycocolle à 20 p. 100 ; tellurite de potassium (B. D. H.) à 1 p. 100 ; pyruvate de sodium à 20 p. 100 .

\section{Préparation du milieu composé}

Au contenu d'une fiole de milieu de base fondu puis maintenu au bain-marie à $45-50^{\circ} \mathrm{C}$, ajouter $6 \mathrm{ml}$ de la solution de glycocolle, $1 \mathrm{ml}$ de la solution de tellurite et $5 \mathrm{ml}$ de la solution de pyruvate. Les solutions sont préalablement portées à $45-50^{\circ} \mathrm{C}$ par séjour de bain-marie.

Ajouter enfin $5 \mathrm{ml}$ d'émulsion concentrée de jaune d'œuf Oxoïd, et compléter, si nécessaire, le volume de milieu à $100 \mathrm{ml}$ avec de l'eau distillée stérile.

Mélanger en agitant doucement, puis distribuer en boîtes de Pétri de $100 \mathrm{~mm}$ de diamètre. 


\section{Ensemencement}

Le milieu est utilisé dans les 24 heures qui suivent sa préparation après que la surface ait été bien séchée par un séjour de 24 heures à l'étuve à $30^{\circ} \mathrm{C}$ ou de 4 heures à $40^{\circ} \mathrm{C}$. Il est ensemencé par étalement uniforme, sur la totalité de sa surface, de $0,1 \mathrm{ml}$ d'inoculum constitué, selon le cas, par le produit brut, le produit dilué, le produit pré-enrichi par incubation ou le produit concentré par centrifugation. Pour remédier aux difficultés de déchiffrement liées à l'envahissement des milieux par un trop grand nombre de colonies non-spécifiques, ou à l'éclaircissement total du milieu dû au développement d'un grand nombre de colonies caractéristiques dont les auréoles transparentes arrivent à confluer en une nappe continue, il est recommandable d'effectuer les ensemencements à deux niveaux différents de dilution : par exemple pour les fromages frais et les crèmes $0,1 \mathrm{ml}$ des dilutions à $1 / 3$ et $1 / 30$; pour le lait cru de mélange $0,1 \mathrm{ml}$ de lait non-dilué et $0,1 \mathrm{ml}$ de dilution à $1 / 10$; pour les laits crus individuels, dans le cas du dépistage de la mammite staphylococcique $0,1 \mathrm{ml}$ de lait non-dilué et, le lendemain, si nécessaire, $0,1 \mathrm{ml}$ de lait pré-enrichi par incubation de 24 heures à 370 , cette pré-incubation pouvant être réalisée sous la forme d'un test de Hotis et Miller ce qui permet éventuellement de détecter aussi Streptococcus agalactiae; enfin, pour les laits individuels, dans le cas du contrôle d'un traitement anti-mammite : $0,1 \mathrm{ml}$ de lait non-dilué et $0,1 \mathrm{ml}$ de culot de centrifugation.

\section{Déchiffrement des résultats}

Les cultures sont examinées après incubation à $37^{\circ} \mathrm{C}$ pendant 24 heures.

Les colonies noires ourlées de blanc, convexes, brillantes, de 1 à $1,5 \mathrm{~mm}$ de diamètre, entourées d'une couronne transparente de 2 à $5 \mathrm{~mm}$ de large tranchant nettement sur le fond opaque du milieu sont comptées pour autant de staphylocoques à DNase positive; ces colonies sont pointées à l'encre : quel que soit leur aspect lors de la seconde lecture, ces colonies seront définitivement enregistrées comme DNase-positives. Les cultures sont de nouveau placées à l'étuve à $37^{\circ}$ et une seconde lecture est effectuée après 18 à 24 heures. Cette seconde lecture permet de détecter des colonies de staphylocoques à DNase-positive qui tardent à s'entourer de l'auréole transparente révélatrice. De telles souches sont fréquemment rencontrées parmi les staphylocoques responsables de cas de mammite de la vache et, par conséquent, leur présence dans les laits crus de mélange restreint et dans les fromages frais de fabrication domestique n'est pas pour surprendre.

C'est ainsi que sur une série d'analyses portant sur 40 échantillons de laits crus individuels ou de mélange restreint, les cultures 
sur milieu de Baird-Parker nous ont révélé la présence, après 24 heures d'incubation, de staphylocoques à DNase positive dans 11 échantillons; une seconde lecture effectuée 24 heures plus tard montrait le développement de colonies tardives à DNase positive dans 5 des 11 cultures déjà comptées comme positives et, en outre, l'apparition de colonies caractéristiques dans 13 autres cultures qui n'en contenaient aucune au terme des premières 24 heures d'incubation. La seconde lecture avait done eu pour effet de porter de 11 à 24 le nombre d'échantillons positifs.

Au total la première lecture donnait 109 colonies de staphylocoques présumés à DNase positive dont 96 p. 100 étaient effectivement confirmées comme DNase-positives; la seconde lecture révélait 213 colonies tardives de staphylocoques présumés à DNasepositive dont 83 p. 100 étaient effectivement confirmées comme DNase-positives.

Les taux respectifs de confirmation par l'épreuve de la DNase font apparaître une spécificité nettement supérieure en faveur des colonies précoces, mais le développement de colonies tardives authentiquement DNase-positives est un fait si fréquent qu'il n'est pas possible de les récuser en dépit de leur spécificité moindre, au moins en ce qui concerne les analyses des laits crus de mélange restreint, et de crèmes et de fromages frais dérivés de ces laits. Il serait souhaitable que la seconde lecture pût être faite 18 heures après la première mais les nécessités des horaires de travail obligent souvent à prolonger ce délai jusqu'à 24 heures, aux dépens de la sécurité de l'interprétation. En tout état de cause, ces 24 heures de seconde incubation marquent le terme extrême au delà duquel les colonies noires entourées d'une large zone transparente ne pourraient plus être comptées comme des staphylocoques à DNase positive, sans qu'une épreuve enzymatique de confirmation leur fût appliquée, mais employé dans ces conditions le milieu de BairdParker ne présenterait plus aucun avantage sur l'ancienne gélose hypersalée au mannitol.

\section{Comparaison entre les résultats des cultures sur mannitol salt agar (MSA) et sur milieu de Baird-Parker (BP)}

La confrontation de deux méthodes a porté : d'une part sur 40 échantillons de lait eru à savoir, 7 échantillons de laits individuels prélevés aseptiquement au pis de la vache et 33 échantillons de laits de mélange restreint provenant d'étables de 6 à 15 vaches ; et d'autre part, sur 22 échantillons de crème ou de fromages frais pasteurisés ou non-pasteurisés (les échantillons trouvés positifs appartenaient tous à la catégorie des produits non-pasteurisés ou incorrectement pasteurisés). Les colonies suspectes ont été soumises à l'épreuve de confirmation de la DNase. La notation d'un résultat positif signifie qu'au moins une colonie caractéristique 
s'était développée sur le milieu considéré et avait été confirmée par le test de la DNase. Nous avons enregistré les résultats suivants :

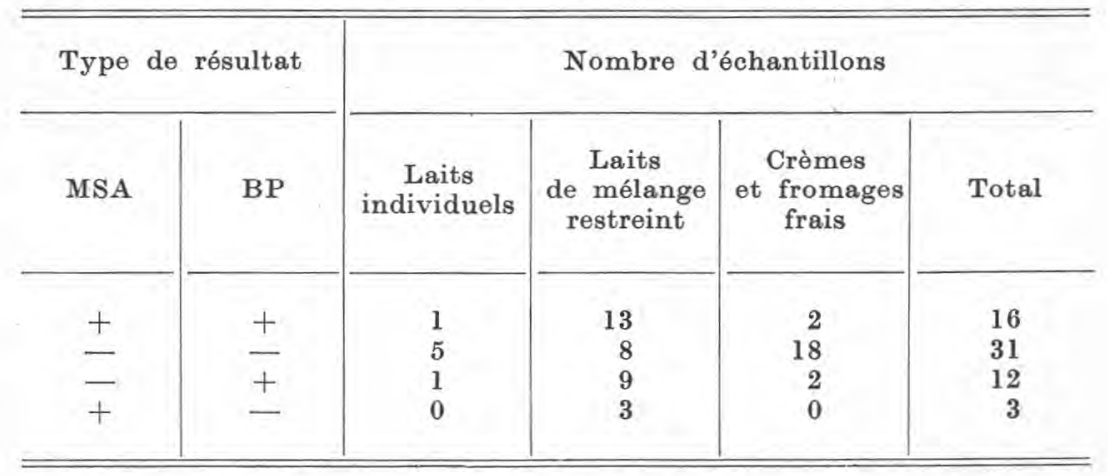

Nous notons des réponses concordantes dans 76 p. 100 des cas, dont 26 p. 100 de cas positifs et 50 p. 100 de cas négatifs.

Nous observons des réponses discordantes dans 24 p. 100 des cas dont 19 p. 100 à l'avantage du milieu de Baird-Parker contre 5 p. 100 à l'avantage de la gélose hypersalée au mannitol.

\section{Conclusion}

Le milieu de Baird-Parker apporte une solution pratique valable au problème du dénombrement direct des staphylocoques à DNase positive du lait et des produits laitiers.

Le taux de confirmation par l'épreuve de la désoxyribonucléase est de 96 p. 100 sur les colonies précoces (incubation 24 heures), et de 83 p. 100 sur les colonies tardives (incubation 48 heures), présentant sur le milieu de Baird-Parker, l'aspect caractéristique (pigmentation noire et large auréole transparente). Le même taux n'est que de 45 p. 100 sur les colonies suspectes développées sur gélose hypersalée au mannitol.

Deux précautions semblent nécessaires :

- Effectuer une double lecture, la première après 24 heures d'incubation à $37^{\circ}$, la seconde de 18 à 24 heures plus tard après nouvelle incubation de façon à dénombrer les colonies DNasepositives qui ne prennent que tardivement leur aspect caractéristique.

- Effectuer la recherche et le dénombrement à deux taux différents de dilution pour éviter d'une part la difficulté d'interprétation liée à la présence d'un trop' grand nombre de colonies caractéristiques dont les auréoles transparentes arrivent à confluer, provoquant un éclaircissement total du milieu sans zones délimitées, et d'autre part l'envahissement du milieu par un trop grand nombre de colonies non-caractéristiques, formant un fond opaque très dense sur lequel il est parfois difficile de distinguer les zones trans- 
parentes, entourant les colonies caractéristiques. Les colonies parasites sont généralement fort nombreuses dans les cultures ensemencées avec des échantillons de lait cru et de produits laitiers non-pasteurisés. Un milieu un peu plus sélectif serait certainement préférable dans ce secteur d'application mais la recherche d'une modification, si minime soit-elle, poserait de nouveau, dans son entier, le problème du dosage des inhibiteurs et des incitateurs en vue de réaliser le périlleux équilibre entre les actions antagonistes de ces composants.

(Laboratoires du Service vétérinaire sanitaire de Paris et du département de la Seine)

\section{Summary}

Baird-Parker's agar enables to number directly pathogenic staphylococcus in milk and milk products.

With "desoxyribonuclease test ", used as a confirmatory test, the proportion of confirmed colonies amounts to $96 \mathrm{p} .100$ for early bacterial colonies (24 hours' incubation) and 83 p. 100 for late colonies (48 hours' incubation) which schowed the characteristic aspect on Baird-Parker's agar (a black pigmentation and a wide transparent halation). The same rate is only 43 p. 100 on doubtful colonies which developped on "mannitol salt agar".

Two precautionary measures, however, seem to be necessary :

1) to take a first reading after 24 hours and a second one after 48 hours.

2) to carry out experiments and counts within two different dilution rates.

\section{RÉFÉRENCES BIBLIOGRAPHIQUES}

BAIRD-PARker A. C. (1962). - J. appl. Bact. 25, 12 et 441.

Barber M. et Kuper S. W. A. (1951). - J. Path. Bact. 63, 65.

Basille D., Pantaleon J,, Rosset R., Gandon Y. et Petit A. (1964). Rapport sur les opérations du Serv. Vet. de la Préfecture de Police en 1963,92 .

Chapman G. H. (1945). - J. Bact. 50, 201 et (1946), J. Bact. 51, 409.

Colbeck J. C. (1956). - Canada Serv. Med. J. 12, 363.

CowAN S. T. (1962). - J. appl. Bact. 25, 324.

Finegold S. M. et Sweeny E. E. (1961). - J. Bact. 81, 636.

Hopton J. (1961), - J. appl. Bact. 24, 121.

INNES A. G. (1961). - J. appl. Bact. 24, 121.

Косн (1942). - Zent. Balt. 149, 122.

Me Culloch (1947). - Am. J. Vet. Res. 8, 173.

NeWMaN (1950). - J. Milk and Food Techn. 13, 226.

Richou R., Pantaleon J. et Quinchon C. (1959). - Annali Sclavo, 1, 647. Sevel B. et Plommet M. (1960). - Le Lait, 40, 2.

Sharpe E., Neave F. K. et Reiter B. (1962). - J. appl. Bact. 25, 403. WeCkMan B. A. et Cathin B. W. (1957). - J. Bact. 73, 747.

Zebovitz E., Evans J. B. et Niven C. F. (1955). - J. Bact. 70, 686. 